\title{
LAZARSFELD IN MEMORIAM. THE PEOPLE'S CHOICE Y VO'TING CUMPLEN SESENTA Y CINCUENTA AÑOS.
}

\author{
JUAN IGNACIO ROSPIR \\ Profesor Titular de Opinión Pública. Universidad Complutense de Madrid
}

\section{Resumen}

La lectura reposada de Lazarsfeld nos devuelve, una vez más, al mundo de innovación, improvisación y sorpresa, en el que algunas de las teorías más conocidas fueron descubiertas y descritas. De "los líderes de opinión" sigue sin estar claro quien fue el progenitor de la idea. Sobre The People's Choice seguimos sin saber por qué se realizó y la comunicación "en dos etapas" continúa siendo un hallazgo inesperado. La relectura de las obras escogidas, una vez más, nos lleva al concepto de "serendipity" como el motor de la investigación en comunicación de masas.

Palabras clave

Lazarsfeld revisitado, encuesta panel, estudios electorales, hallazgos inesperados.

Abstract

A careful reading of Lazarsfeld sends us back, once again, to the world of innovation, improvisation and surprise, a world in which some of the best-known theories were discovered and described. It is still not clear who originated the idea of "opinion leaders". We still don't know why The People's Choice came about, and "two stage communication" is still an unexpected discovery. The re-reading of these chosen works, once again, returns us to the concept of "serendipity" as the engine behind mass communication research.

Key Words

Lazarsfeld revisited, panel survey, electoral studies, unexpected discoveries. 


\section{INTRODUCCIÓN}

In Memoriam. El año 2002, con más discreción que alborozo, se ha recordado el centenario del nacimiento de Paul Felix Lazarsfeld. En el año 2004 se cumplen sesenta y cincuenta años de la publicación de The People's Choice (1944) y de Voting (1954) respectivamente. Ambas publicaciones forman parte del patrimonio académico que configura la tradición de investigaciones electorales en opinión pública, comunicación de masas y sociología política. La relectura hoy de ambas obras nos devuelve a la génesis de conceptos tan conocidos y revisitados en comunicación de masas como el de "líderes de opinión", "la encuesta panel" o los "efectos limitados de los medios". Todos ellos son conceptos explicados en un gran número de manuales y textos ad hoc empleados en los departamentos de periodismo o comunicación. Sin embargo, nuestro propósito es recordar cómo estos "descubrimientos" fueron inesperados, incompletos $y$, más relevante todavía, sin que los autores recuerden las causas o motivos que les llevaron a realizar la investigación caso, por ejemplo, de The People's Choice. Pasados los años también hemos sabido que un concepto tan gravitatorio en la sociología de la comunicación como el de "líderes de opinión", su autor, Lazarsfeld, reconoció que la idea original de este modelo comunicativo no era suya. Del trabajo de campo y sus responsables sería injusto seguir olvidando a colaboradores como Elmo Roper y el modelo de relación profesional que se estableció. Lazarsfeld supo unir a los pollsters y a la academia en un estilo de trabajo que hoy es reconocido como otra de sus aportaciones.

Recobrar una vez más la importancia de Paul Felix Lazarsfeld para el estudio de la opinión pública es subrayar, desde el peligro constante que supone hundirse en su extensa y variada obra, el significado y la expansión que sus constantes innovaciones metodológicas trajeron a la explicación e investigación de la formación y difusión de la opinión pública en su expresión numérica o cuantitativa. Un significado que se acrecienta paulatinamente a medida que se recorre su dilatada carrera académica a través de sus publicaciones, su preocupación por clarificar el lenguaje conceptual o su incesante labor como creador de centros de investigación. Actividades que han dejado una huella intelectual indeleble, a pesar de que no tengamos una teoría con T mayúscula de Lazarsfeld como progenitor, pero sí una constante referencia a su figura académica que engrandece su aportación.

Su rigor e innovación metodológica junto con su lenguaje científico, en un tiempo en que la euforia funcionalista y empírica oscureció tantas explicaciones y debates teóricos, no fue obstáculo para que en opinión pública y, en un sentido más amplio, en comunicación de masas, debamos a Lazarsfeld algunos de los cambios y teorías más conocidas y aceptadas durante más de dos décadas. Sus aportaciones al cuestionario, la entrevista, el muestreo, el análisis secundario, el estudio de casos, la encuesta panel, los "líderes de opinión" y "la comunicación en dos etapas", constituyen hoy un capítulo propio del estudio de la opinión pública a pesar de no estar interesado su autor ni por la cuantificación ni por la comunicación per se. La influencia que irradió, como creador institucional, a través de su famoso Bureau Applied Social Research (BASR) y sus numerosos colaboradores, amigos y discípulos, constituye igualmente otro importante apartado de la institucionalización de la investigación en opinión pública. Para una correcta comprensión de lo que Lazarsfeld significa en nuestro campo de conocimiento los criterios habituales de exposición han si- 
do el cronológico y el temático. Las fuentes para ambos criterios, como es fácil suponer, han sido las propias obras del autor, su "Memoria" académica, así como las biografías y memorias escritas por sus amigos, colaboradores y discípulos más conocidos. Los testimonios de estos últimos, a medida que pasa el tiempo, se han convertido en el complemento indispensable para conocer el estilo de trabajo diario, los problemas financieros que lo rodeaban o la convivencia académica que se producía. Unas fuentes de información, en suma, que hace más atractivo y real el conocimiento de nuestro autor'.

La vinculación de Lazarsfeld con la opinión pública se puede referir, como criterio básico de ordenación, a través de los tres principales campos de interés que muestra su obra: los problemas e innovaciones metodológicas; la orientación histórica y la aplicación de los métodos. Sin embargo, hemos preferido tomar la clasificación que James S. Coleman reconoce como los "seis comienzos" para explicar el cambio de dirección que Lazarsfeld introdujo en la sociología: 1) la transformación de los sondeos de opinión en investigación de encuestas; 2) el comienzo de la técnica de la encuesta panel; 3) el estudio sistemático de audiencias, comunicación de masas e investigación de mercado; 4) el comienzo del método de encuesta en la sociología política (estudios electorales); 5) el desarrollo de la moderna sociología matemática, y 6) la creación de los centros de investigación social aplicada en la Universidad (Coleman, 1982: 2). De estos "seis comienzos" los cuatro primeros son los que más directamente atienden o afectan el desarrollo de la comunicación de masas.

Los dos primeros pertenecen al triunfo de la cuantificación en opinión pública sin cuya referencia es difícil entablar su complejidad y desarrollo posteriores. Como afirma J. S. Coleman: "Lazarsfeld fue de la mayor importancia en la transformación de los métodos de los sondeos de opinión en análisis de encuesta, esto es, dentro del uso analítico de las encuestas por muestreo para sacar inferencias acerca de relaciones causales que afectan la acción de los individuos. Es difícil imaginar la sociología sin tales análisis" (Coleman, 1982: 23). La encuesta panel es la otra gran transformación que para Coleman introdujo Lazarsfeld en los sondeos de opinión. Charles Y. Glock, por su parte, destaca cómo en su forma primitiva todos los ingredientes de la moderna investigación de encuesta estaban presentes en el tiempo en que Lazarsfeld empezó su trabajo en Viena. La identificación de Lazarsfeld como padre de la moderna investigación de encuesta, Glock la resume con estas palabras: "Proviene por tanto no de haber sido el inventor de sus componentes sino de haberlos puesto a ellos juntos en una forma única" (Merton, Coleman, Rossi, 1979: 23). La sín-

\footnotetext{
' Lazarsfeld, P. F. (1969): "An Episode in the history of Social Research: A Memoir by Paul Lazarsfeld" en, Fleming, D. y Bilying, B. (eds.): Intelectual Migration: Europe and America, 1936-1960, Cambridge: Harvard University Press, pp. 270-337. Traducción al español, Jiménez Blanco (2001): "Memoria de un episodio en la historia de la investigación social", REIS, 96, pp. 235-296. Merton, R. K. (1979): "Remembering Paul Lazarsfeld", en Merton, R., Coleman, J. y Rossi, P. (eds.): Qualitative and Quantitative Social Resarch. Papers in honor of Paul F. Lazarsfeld, New York: The Free Press, pp. 19-23. Coleman, J. S. (1993): "La Universidad de Columbia en los años cincuenta", en Berget, B. M., La sociología como profesión. Autobiografía intelectual de veinte sociólogos, Madrid, Ministerio de Trabajo, pp. 137-176. Noelle-Neumann, E. (2001): "My Friend, Paul Lazarsfeld", International Journal of Public Opinion Research (IJPOR), 13, 3, pp. $315-326$.
} 
tesis que hizo Lazarsfeld, según este autor, es la que más ha hecho por que el método de investigación de encuesta sea el predominante en la investigación social. El tercero y cuarto de los puntos considerados por Coleman le atribuyen el reconocimiento de "padre fundador" del estudio moderno de la comunicación de masas en un tiempo en que la sociología estudiaba dicha comunicación en relación con la opinión pública. Los estudios electorales realizados en 1940, The People's Choice, y 1948, Voting, trajeron una serie de asunciones que hoy nos resultan habituales pero que en aquel entonces apenas se realizaban. Paul Lazarsfeld, hay que recordar, abandonó el campo de la comunicación de masas, como preocupación y dedicación académica, en 1956. Un momento (1956-1960) que es reconocido como el final de una de las etapas de la historia de la comunicación de masas. Con el abandono de Lazarsfeld y de otros sociólogos los objetivos recogidos en su conocido artículo conjunto con Robert Merton, "Mass Communication, popular taste, and organized social action"2, dejaron de estudiarse para dar paso a una nueva etapa, más estrictamente comunicacional, de los nuevos problemas y objetivos señalados (Berelson, 1959: 1-17; Katz, 1987: 22-45). Lazarsfeld anunció su "adiós" declarando que no dirigiría ninguna tesis doctoral más sobre comunicación de masas (Schramm, 1997). La última fue la de Elihu Katz realizada a partir de su interpretación y codificación de los datos recogidos en la elaboración del conocido estudio, La influencia personal (1955).

\section{THE PEOPLE'S CHOICE}

La investigación realizada en Eire Country (Ohio) en 1940 a partir de la campaña presidencial que enfrentó a F. D. Roosevelt con Wendell L. Willkie, dio lugar al que seguramente es el libro más conocido de Lazarsfeld en el ámbito de la opinión pública y la comunicación de masas: The People's Choice. How the Voter Makes Up His Mind in a Presidential Campaign (Lazarsfeld, Berelson y Gaudet, 1944). Dicha obra abrió una nueva visión de la campaña electoral y creó un "banco de pruebas" sobre y contra el cual hoy seguimos discutiendo. El origen y los motivos de tan citada obra todavía hoy los desconocemos. Por la "Memoria" de Lazarsfeld sabemos que a principios de 1940 obtuvo una nueva financiación de la Fundación Rockefeller para estudiar por tres años, con la técnica del panel, los efectos de la radio en los cambios de los hábitos de comportamiento diario. Sin embargo, como escribe el autor: "Yo no recuerdo cómo esta propuesta fue alterada, así que las elecciones de 1940 se convirtieron en el centro del primer estudio de panel". Otros colaboradores de aquel momento confirman esta versión y tampoco recuerdan los motivos que propiciaron el cambio. The People's Choice es la obra en que se presenta por primera vez plenamente el potencial que la técnica de la encuesta panel encerraba para el estudio de los efectos de la propaganda en el cambio de opinión e intención de voto. En la investigación, además de la Fundación Rockefeller y la Office of Radio Research, Elmo Roper participó como patrocinador con-

\footnotetext{
${ }^{2}$ Lazarsfeld, P. F. y Merton, R. (1948): "Mass Communication, popular taste, and organized social action" en, Bryson, L. (ed.): Communication of Ideas, New York: Institute for Religious and Social Studies, pp. 95-1 18. Reproducido en Schramm, W. (1971): The Process and Effects of Mass Communication, Urbana Illinois: University of Illinois Press, pp. 554-578. Traducción al español, Moragas, M. (ed.) (1979): Sociología de la comunicación de masas, Barcelona: Gustavo Gili, pp. 137-157.
} 
tribuyendo con su equipo y experiencia encuestadora. Las revistas Life y Fortune, a cambio de publicar durante la campaña algunos avances de los datos de la investigación, también aportaron fondos. Las dificultades para financiar la investigación Lazarsfeld las desveló en 1957, aportando cifras concretas, con motivo del XX aniversario del BASR (Converse, 1987: 271). El libro se publicó en 1944, el año en que la Office of Radio Research es renombrada como el Bureau of Applied Social Research (BASR), y es a partir de esa fecha cuando se empieza a extender en el ámbito académico la técnica del panel empleada cuatro años antes. Empieza lo que su investigador principal bautizó como "la tradición de Columbia".

La encuesta panel no es un invento de Lazarsfeld ni esta era la primera vez que se aplicaba, pero sí la primera vez que se ejecutaba en la forma en que después se ha generalizado. Experiencias anteriores con estudiantes y lectores de revistas se habían realizado en los años veinte. En los treinta, se realizaron las primeras aplicaciones con oyentes de radio dentro de la investigación de mercado. Las de carácter político, también en los años treinta, 1938, fueron dos: una referida a la elección del Gobernador de Nueva York y otra con motivo de un referéndum en Pennsylvania para estudiar los cambios de opinión y los efectos de la campaña. En esta época la encuesta panel se realizaba a través de cuestionario por correo. De estos precedentes los trabajos más conocidos son los de S. Rice, "Quantitative Methods in Politic" (1929) y el de T. Newcombs, "Personality and Social Change" (1943). Lazarsfeld también había mostrado su interés por el panel -que nunca abandonó- en dos artículos anteriores. Uno de 1938, "The 'Panel' as a new tool for measurimg opinion", en colaboración con M. Fiske (Lazarsfeld y Fiske, 1938: 596-612) y otro, en 1940, titulado, "Panel studies" (Lazarsfeld, 1940: 122-128). En el primero ya expone los motivos de sus ventajas y los beneficios que representa. Cita los precedentes más relevantes a la vez que recoge y señala como el principal inconveniente, no la mortalidad de la muestra, sino "el efecto panel", es decir, el compromiso y la implicación que desarrollan los miembros que en él participan. En el segundo, marzo de 1940, es decir, antes de comenzar en Eire Country, empieza por señalar el creciente interés que las empresas de sondeos de opinión pública tenían en la técnica panel para estudiar los efectos de las campañas comerciales. Es un comienzo de cuya lectura se desprende que Lazarsfeld en ese momento identifica la opinión pública con los sondeos y su técnica. Los datos de un estudio de 1938 realizado por la Office of Radio, con 358 personas, le sirven para comentar la fiabilidad estadística, el coste comercial, su adecuación para el estudio de los efectos acumulados, el problema de la admisión de los cambios por parte de la muestra, el grado de cooperación, y concluye afirmando: "El panel es el instrumento más válido en el estudio de los efectos de la propaganda". El conjunto de publicaciones que dedica entre 1940 y 1972 a esta técnica es la mejor prueba de su interés por la misma. La novedad que se incorpora en The People's Choice es la aplicación por primera vez de la técnica panel a una muestra representativa de la población. Como señala Allen Barton, director del BASR entre 1962 y 1977 y ayudante de Lazarsfeld, con la técnica del panel aplicada en Eire Country, "fue posible estudiar el proceso dinámico de la decisión individual, como influida por las comunicaciones de la campaña y por los procesos psicológicos y sociales realzados por la misma" (Barton, 2001: 253). En el contexto histórico que estamos tratando el uso del panel realizado por Lazarsfeld fue calificado por Herbert Hyman, en un tiempo también colaborador, como "su más notable contribución a la investigación de encuestas" (Hyman, 1991: 155). 
La explicación de cómo se desarrolló la técnica del panel en el estudio de 1940 la describe Lazarsfeld en el "Prefacio" a la tercera edición publicada en 1968. Antes, en la "Introducción" a la primera edición de 1944, ya había dejado claro algo sugerido en su artículo de noviembre de 1940: las limitaciones de los sondeos de opinión para este tipo de estudios. "El pleno efecto de una campaña-dice ahora- no puede ser investigado a través de una secuencia de sondeos realizados con diferentes personas. Muestran solamente tendencias mayoritarias que actualmente son el resultado residual de varias clases de cambios, o desde la indecisión o desde un partido a otro". En la investigación que ha emprendido, dirá Lazarsfeld, los sondeos "no muestran quién está cambiando. No siguen los caprichos del votante individual a lo largo del patrón de su voto, para descubrir el efecto relativo de los varios factores influyentes sobre su voto final". En mayo de 1940, una de cada cuatro casas de Eire Country fue visitada por un miembro del equipo de E. Roper; en total fueron entre 12 y 15 mujeres las entrevistadoras, para "aproximadamente -dice Lazarsfeldelegir 3.000 personas representativas del conjunto de la población del condado. De estos 3.000, por muestreo estratificado, se sacaron cuatro grupos de 600 personas. De estos cuatro grupos, tres fueron reentrevistados una sola vez, agosto y octubre, y utilizados como grupo de control para estudiar los efectos que la repetición de las entrevistas podía tener. A la vez, también se extrajo de ellos otra muestra de 1.200 para realizar preguntas puntuales de control. El cuarto grupo de 600 constituyó el panel. Fue entrevistado una vez al mes desde mayo a noviembre. Las entrevistas se espaciaron por meses para seguir y recoger mejor la campaña electoral: nominaciones, convenciones, etc. La última entrevista al panel, noviembre, se hizo después de las elecciones.

La técnica del panel es presentada por Lazarsfeld en la "Introducción" como la más adecuada para conocer qué efectos tiene el estatus social sobre el voto, cómo influyen las nominaciones y convenciones de los partidos, qué papel juega la propaganda, cómo actúan la prensa y la radio, qué influencia tienen la familia y los amigos, cómo surgen los temas de campaña, por qué algunas personas deciden su voto al principio y otras al final, en suma, cómo se desarrolla la intención de voto y por qué. Al conjunto de todas estas preguntas es a lo que The People's Choice responde a lo largo de sus 16 capítulos. No es, por tanto, como frecuentemente se cree, una investigación y un libro concebidos únicamente para estudiar el proceso de la comunicación de masas en una campaña electoral. El interés principal radicó en conocer cómo se desarrollaba la intención de voto y las causas de su decisión final. Dentro de estos objetivos una parte de la investigación estaba pensada para conocer el papel que juega la propaganda durante la campaña.

Las conocidas teorías de los "líderes de opinión" y "la comunicación en dos etapas" son tratadas en el conjunto de los capítulos $\mathrm{V}$ y XVI. La primera con una extensión de tres páginas y la segunda dentro del concepto más amplio de la naturaleza de la influencia personal. El capítulo XVI es el que cierra el libro y fue añadido al final de la investigación. En el "Prefacio" a la edición de 1968 Lazarsfeld escribe: "La comunicación persona a persona, nos sorprendió en el transcurso de la investigación. El estudio -continúa- no nos permitió documentar esta impresión muy especialmente porque nuestro esfuerzo principal estaba dirigido a establecer el papel más formal de los medios de comunicación. Todo lo que nosotros pudimos hacer en aquel tiempo fue sacar un número de especulaciones, girando acerca de los líderes de opinión que habíamos identificado". Más adelante, para aclara este comentario. escribe: "Aunque nosotros no examinamos a fondo la natura- 
leza de la influencia personal en el estudio de 1940, nosotros aludimos a la concepción de un 'flujo de la comunicación en dos etapas". En 1972, cuatro años antes de morir en 1976, vuelve a escribir: "The People's Choice, un estudio del papel de los mass media en la campaña presidencial de 1940, me hizo sentir que la influencia personal jugó un gran papel en la forma que la gente realiza su decisión de voto. No habiendo anticipado este tema yo no tuve datos par seguir la pista cuantitativamente. Por tanto uní a nuestro libro un capítulo sobre 'La naturaleza de la influencia personal' el cual se basó solamente en unas pocas observaciones cualitativas" (Lazarsfeld, 1972: 13). Ambos descubrimientos serán analizados más detenidamente en las investigaciones posteriores de Voting y La influencia personal. Sí hay que decir que con la materia prima obtenida en esta primera investigación se publicaron posteriormente varios trabajos monográficos dedicados a profundizar en el perfeccionamiento de la metodología empleada y sus hallazgos. De los discípulos y colaboradores que se dedicaron a esta tarea, Lazarsfeld da cumplida cuenta en el "Prefacio" de 1968. La segunda edición de 1948 también contiene un "Prefacio" en el que Lazarsfeld comenta los aspectos metodológicos de la obra y reclama una integración entre la investigación empírica y la teoría social. Plantea la necesidad de un marco teórico más amplio e integrador para el conjunto de los datos obtenidos. Su propuesta la explicita en una lista de ocho apartados en la que formula el camino que hay que seguir para dicha investigación. Los tres últimos apartados son los dedicados al campo de la comunicación de masas e interpersonal. La conveniencia de repetir la investigación de 1940 en un marco histórico y político distinto también es sugerida lo mismo que la necesidad de perfeccionar la técnica del panel.

En The People's Choice la opinión pública no aparece definida ni explicada en ningún capítulo de la obra. Es en el "Prefacio" a la segunda edición de 1948 donde Lazarsfeld formula algunas aclaraciones y comentarios que relacionan la investigación realizada con el concepto de opinión pública que él considera. Es el epígrafe titulado, "Dinámica de la investigación social". Empieza por afirmar que la investigación de la opinión pública es frecuentemente mal entendida. Desde los datos de los sondeos publicados en revistas y periódicos, legos y expertos de otros campos de las ciencias sociales han llegado a la impresión de que tal investigación está circunscrita a describir qué siente la gente sobre un tema dado en un momento concreto. "Actualmente -continúa- el ámbito de la nueva disciplina es mucho más amplio. Los científicos sociales quieren conocer los procesos por los cuales varios sectores de la opinión pública influyen en la acción legislativa y en otras decisiones del gobierno. Además, nosotros estamos deseosos por descubrir en qué forma las actitudes mismas se forman. The People's Choice centró su atención sobre este segundo problema, la formación, cambio y desarrollo de la opinión pública". Con la designación de ambos problemas -los procesos de influencia de la opinión pública y la formación y cambio de actitudescomo nuevo ámbito de la opinión pública, Lazarsfeld reconoce y empelle los nuevos objetivos y el camino metodológico por el que la opinión pública empieza a desarrollarse en esta nueva etapa. El avance metodológico impulsado por Lazarsfeld es sin duda el que situó a la opinión pública en la investigación cuantitativa de la formación y cambio de actitudes.

Los resultados del estudio fueron inesperados. No hay que olvidar que la investigación está realizada en los años de la Office of Radio Research lo que significa un interés por conocer la influencia de la radio, sus efectos directos, durante la campaña electoral. Insospechadamente, los re- 
sultados revelaban una influencia modesta de este medio y la influencia más importante de la comunicación interpersonal, es decir, la mediación de los líderes de opinión en el flujo de la influencia entre los medios y los ciudadanos. Los hallazgos principales -los efectos de las presiones cruzadas sobre la decisión de voto, los "líderes de opinión" y la "comunicación en dos etapas"aunque en un primer momento se entendieron como teorías de "rango medio", así aparecen en el Prefacio a la segunda edición de 1948, la atención que atrajeron y las investigaciones que se sucedieron los convertirán en generalizaciones casi indiscutibles durante más de veinte años siendo estudiadas y conocidas como "el modelo de efectos limitados". Steven H. Chaffe y J. L. Hochheimer han señalado cómo en la encuesta postelectoral -noviembre- que Lazarsfeld realizó para volver a preguntar por las fuentes de información que habían conducido a la decisión final, "más de dos tercios -subrayan estos autores-mencionaron periódicos y emisoras de radio como la fuente más útil, mientras menos de la mitad mencionó algún tipo de contacto personal" (Chaffé y Hochheimer, 1985: 75-104). Este comentario, sin embargo, no aclara que Lazarsfeld en este epígrafe de la obra (páginas 125-127), y así se reproduce en las gráficas, estaba diferenciando entre "más útil" y "más importante". Los críticos y discrepantes con este modelo se centrarán en denunciar el contexto político de la campaña, su metodología y las extrapolaciones de los datos a otras situaciones electorales y políticas (Rospir, 1991: 280-299).

\section{VOTING}

El segundo gran estudio electoral dirigido por Lazarsfeld en colaboración con Bernad Berelson y W. N. McPhee fue, Voting. A Study of Opinion Formation in a Presidencial Campaign (1952). Es una replicación aproximada de la investigación anterior de 1940 y está realizada en el Condado de Elmira (Nueva York) durante la campaña presidencial de 1948 que enfrentó a $H$. Truman y Thomas E. Dewey. Está, por tanto, dentro del conjunto más amplio de estudios electorales realizados entre 1940 y 1952 -cinco en Estados Unidos y dos en Gran Bretaña- que dieron lugar a un conjunto de 14 publicaciones aparecidas entre 1941 y 1954. Voting se publicó en 1954, lo que significa que antes de su aparición ya se conocían, al menos, siete publicaciones que hubiesen aplicado también la técnica del panel. Fuera de estos estudios electorales, La influencia personal, aunque publicada en 1955, sus experiencias del trabajo de campo realizado en 1945 pudieron incorporarse. Voting fue un estudio sobre cómo la influencia de los procesos sociales transforma las intenciones de voto en una decisión final. Es una obra más analítica que descriptiva, lo que la diferencia de la anterior de 1940. Combina mecanismos psicológicos y variables sociales para estudiar el cambio de opinión, el entorno interpersonal, las predisposiciones políticas, el papel de los partidos, la activación del interés político y la discusión por la influencia de la comunicación y cómo la influencia personal refuerza las predisposiciones y normas políticas tradicionales. Como escribe Slavko Splichal, "por primera vez se enfrentaron al problema del determinismo social, o la influencia del entorno social y político en la formación de la opinión" (Splichal, 1989: 94).

En la "Introducción" se insiste una vez más en la adecuación de la técnica del panel para analizar los procesos que están desarrollándose y anticipar los elementos psicológicos y sociales 
que pueden jugar un papel en la decisión de voto. El panel es definido como "un método particular de estudio del desarrollo y cambio de opinión entre las mismas personas a lo largo del tiempo". También recoge cómo dicha técnica lleva a introducir variables que no están incluidas en los habituales sondeos de opinión pública. Con anterioridad, noviembre de 1948, cuando el trabajo de campo de Voting está realizándose, Lazarsfeld publicó "The Use of Panels in Social Research"3, una contribución que demuestra su interés y confianza en dicha técnica. En dicho artículo fija las diferencias más importantes entre el panel y los datos de "tendencias" obtenidos a través de sondeos de opinión. Recoge cómo el panel -frente a las tendencias de los sondeos- permite conocer quienes son los que cambian de opinión y profundizar en los factores sociales y psicológicos de dicho cambio. Igualmente, subraya la adecuación del panel para conocer el papel que los medios de comunicación juegan en el cambio de opinión. Reconoce que el panel está en sus comienzos y que necesita de arduos trabajos en el futuro pero manifiesta abiertamente su confianza en su uso para investigar el comportamiento individual.

Para centrar el asunto del libro, la "Introducción" señala cómo The People's Choice reconocía parcialmente la importancia de la influencia personal de unos votantes sobre otros al igual que sin resolver señalaba el problema del papel político que jugaban los "temas" de campaña. "El presente libro -escriben los autores- concede una atención particular a estos dos asuntos. En el caso de la influencia personal, lo que antes fue una materia de conjetura, ahora está documentado y elaborado; en el caso de las cuestiones de campaña, lo que fue un puzle, ahora está empezando a ser aclarado". El libro está organizado en dos partes, la social y la política. La primera está dedicada al estudio de la formación de preferencias y la segunda al comportamiento del electorado. En Voting se confronta la teoría democrática con la práctica democrática revelada por la investigación. En esta confrontación el estudio de los líderes de opinión está dentro de la parte social, capítulo $\mathrm{VI}$, y el papel de los medios de comunicación, capítulo XI, dentro de la política. Los dos últimos capítulos, XIII y XIV, están redactados por Lazarsfeld y Berelson, respectivamente. En el XIII su autor describe su propuesta de explicación psicológica de la decisión de voto y en el XIV, Berelson armoniza la suya desde la confrontación entre teoría y práctica de la democracia revelada por los sondeos.

La ciudad elegida, Elmira, no respondió a las expectativas de "ciudad obrera" consideradas por los autores. El augurio de que los líderes sindicales participarían activamente centrando algunos de los "temas de la campaña" no se cumplió. Elmira era de una tendencia tan republicana que los comités electorales de los sindicatos no enviaron ni representantes ni dinero. Estos datos sirven para ilustrar cómo la fluctuación del voto, prevista por la influencia de los medios o por contactos personales, tenía muy pocas posibilidades de producirse ante el voto consolidado que caracterizaba a la ciudad. La primavera de 1948 fue también la del Plan Marshall para Europa, el bloqueo

\footnotetext{
${ }^{3}$ LAZARSFELD, P. F. (1948): "The Use of Panel in Social Research", Proceeding of the American Philosophical Society, 92, pp. 405-410. Reimpreso en LAZARSFELD, P. F, A. PASANELLA y M ROSEMBERG (Eds.) (1972): Continuities in the Language of Social Research, New York, The Free Press, pp. 330-337.
} 
de Berlín y su famoso "puente aéreo". El comportamiento electoral norteamericano en los años cuarenta y cincuenta, Lazarsfeld lo describió en 1963 en "Political Behavior and Public Opinion"4. Aunque de forma un tanto simplificada, pero adecuada para el conjunto de esos años, resumió los principales argumentos y clasificaciones que sirvieron para interpretar dicho comportamiento. Cuando la golden age de los años cincuenta desaparezca algunas de sus generalizaciones llegarán hasta nosotros convertidas en estereotipos del análisis electoral.

En esta investigación, los autores tuvieron que elegir, por razones presupuestarias, entre utilizar una muestra mayor $y$, por tanto, obtener más respuestas o una cantidad mayor de repeticiones de entrevistas -oleadas de panel- que en 1940. La elección fue obtener más respuestas. El diseño de la muestra fue el probabilístico por áreas, introducido en la década de los cuarenta, para asegurar una mayor selección al azar. Se obtuvieron -el proceso se describe en el Apéndice C- 1.267 hogares en los cuales viviese un adulto. Se realizaron cuatro oleadas de entrevistas: junio con 1.267; agosto con 814; octubre con 881 y noviembre con 944 . La mortandad de la muestra estuvo entorno al $12 \%$. Los cuestionarios de cada oleada y los índices están recogidos íntegros en el Apéndice B. El índice de discusión política consistió de dos preguntas en junio y octubre y el de líderes de opinión de otras dos en junio. Tanto Voting como The People's Choice son las dos investigaciones que en el período de 1940 a 1952 realizaron mayor número de oleadas de panel, cuatro y seis respectivamente. Otras investigaciones aplicaron sus entrevistas una antes y otra después de las elecciones. En algún caso sí utilizaron muestras mayores. Cuando el libro se publica en 1954 la técnica del panel ya es habitual en la investigación en ciencias sociales. Otra aportación significativa de Voting -como más tarde recordará R. K. Merton- fue el uso sistemático del concepto de "grupo de referencia" de Herbert Hyman para el análisis de datos. Concepto y experiencia que conducirá a la idea metodológica del análisis de muestras contextuales. La incorporación de los contextos sociales en el diseño de encuestas la aplicará Lazarsfeld en The Academic Mind (1955) y S. M. Lipset en Union Democracy (1956).

Los "líderes de opinión" en Voting fueron estudiados dentro de una red de relaciones de dirección de la opinión -como un sistema nervioso a través del cuerpo, escriben los autores- que en la práctica constituyen circuitos de liderazgo que están compitiendo en el seno de la comunidad. En dicha red o circuito es posible aislar e identificar a aquellos individuos que están más próximos a ocupar una posición central de relaciones a las que se llama "líderes de opinión". Esta clase de personas, los líderes de opinión, se distinguen por su mayor interés y competencia en el ámbito de la discusión; por una interacción más activa -fruto de su posición estratégica en la comunidad-y por la representatividad de aquellos a quienes influyen. Los líderes de opinión se encuentran en todos los estratos sociales.

El concepto de "líderes de opinión" tan unido a la sociología de la comunicación de Lazarsfeld no tiene, sin embargo, un origen claro en lo que respecta a la génesis de la idea. En Voting

\footnotetext{
${ }^{4}$ LAZARSFELD, P. F. (1963): "Political Behavior and Public Opinion" en BERELSON, B. (Ed): The Behavioral Sciences Today, New York, Basic Books.
} 
son dos las invocaciones que se recogen como precedentes, una en el capítulo VI y otra en el XIII. En el capítulo $\mathrm{VI}$, en el que se trata este concepto, se recoge una cita de J. Bryce, The American Commonwealth, para presentar el epígrafe del mismo. "La opinión -dice Bryce- no solamente se desarrolla, también se fabrica. No hay solamente una clase de personas pasivas, está la clase activa, quienes esencialmente se ocupan de los asuntos públicos, quienes aspiran a crear y dirigir la opinión" (Vol. 2. Cap. 80). En el capítulo XIII, Lazarsfeld recupera lo que llama la tradición de Tarde y expone cómo el estudio de los contactos y las conversaciones personales es parte de su propuesta sociológica, a la vez que denuncia cómo este componente ha sido rechazado durante algún tiempo. La recuperación de Tarde la inicia Lazarsfeld subrayando cómo con el cambio de siglo "la opinión pública dejó de ser un tema de especulación general y se volvió objeto de específicas y detalladas monografías". El texto que señala para este empeño es, lógicamente, el epígrafe "La conversación", correspondiente al capítulo II de su conocida obra La opinión y la multitud. La explicación de Tarde de que la opinión se forma realmente a través del intercambio diario de comentarios y las observaciones que circulan entre las personas y que el estudio empírico de la conversación era básico para la sociología, Lazarsfeld lo califica de "muy próximo" a la propuesta que formula en este capítulo. Más adelante declara cómo cuando The People's Choice fue redactado los autores desconocían estas ideas de Tarde. También explica la diferencia entre lo que denomina la "posición asimétrica" en el estudio de los líderes en 1940 y la "sociología simétrica" que está proponiendo ahora. Los "líderes de opinión" en 1940 se vieron a través de una unidad individual de análisis y no de una colectiva. La atención se centró en los individuos de la muestra y las conversaciones que mantenían con los de fuera (posición asimétrica). "Se vuelve obvio -escribe Lazarsfeld ahora- que no hay diferencias entre personas dentro y fuera de la muestra. [...] La solución correcta es hacer de la conversación -la pareja o el grupo de interlocutores- la unidad de análisis. Esto nos devuelve al punto de partida, al pensamiento que compara las ideas de Tarde y el material recogido en este libro, especialmente en el capítulo VI". Evertt M. Rogers, al ocuparse del origen de este concepto, narra cómo Lazarsfeld reconoció que el concepto de "líderes de opinión" se lo debía a Edward Bernays, padre de las relaciones públicas y autor en 1923 de Cristalizing Public Opinion, a quien conoció en su primer viaje a Estados Unidos en 1933 y con el que mantuvo una larga amistad. Edward Bernays, por su parte, cuenta Rogers citando una entrevista personal con el interesado, le declaró que él lo había tomado a su vez del libro de Walter Lippmann, Public Opinion (1922) (Rogers, 1994: 287).

El Capítulo XI, "Political Processes: The Role of the Mass Media", es el dedicado al papel de los medios de comunicación en el proceso político y el más conocido de esta obra. Por sus múltiples reproducciones y traducciones se ha convertido en un texto clásico de la sociología de la comunicación. Los autores identifican y comentan, a partir de los datos obtenidos, los componentes y la visión de lo que después hemos conocido como el modelo de efectos limitados. Un modelo, hay que recordarlo, que fue elaborado sin la incorporación aún de la televisión como medio de comunicación de masas, lo que explica que únicamente aparezcan tres referencias a dicho medio sin alusión a ningún tipo de datos. Los autores empiezan por señalar cómo los medios de comunicación amplían el debate político más allá de las relaciones cara a cara y cómo el electorado es parte de un sistema de discusión. La manera en que se tramite la información política por "agentes no neutrales" es calificada como un "problema de envergadura" para las democracias contemporá- 
neas. El concepto de exposición a los medios es el centro de este capítulo. Empiezan por admitir -igual que hoy- que es una cuestión de "definición arbitraria" y se pasa a establecer lo que conocemos como los "determinantes de la exposición". Diferencian dos tipos de exposición: 1) la intensa, la de aquellos que atienden y tienen interés en la campaña; y 2) la moderada, los muchos que "también están presentes". La exposición es reconocida como la función de clarificar las percepciones políticas. La explicación de su difusión en la comunidad es explicada a través del "factor general de la presión social", entendida como el entorno social y las expectativas interiorizadas del individuo respecto a su comportamiento en el grupo y en la comunidad. La audiencia de las campañas políticas es considerada por los autores una mezcla de la "minoría" interesada y la "mayoría" que está presente. Los efectos de los medios durante la campaña quedan resumidos en la frase más conocida de este capítulo: "La exposición a los medios de comunicación provoca el voto al propio tiempo que solidifica preferencias. Cristaliza y refuerza más que convierte".

El libro concluye con el capítulo de Bernad Berelson dedicado a la confrontación entre requerimientos de la democracia -discusión, interés, motivación, conocimiento, racionalidad, individualidad-y los hábitos y pautas de comportamiento electoral encontrados en la investigación y colocados en el ciudadano medio. A partir de varias citas de James Bryce, Berelson describe las diferencias y la distancia que la investigación emprendida muestra en el funcionamiento de los medios de comunicación, los cambios de opinión y la decisión de voto respecto de la teoría aceptada. Este mismo tema lo había adelantado ya en su artículo de 1952, "Democracy Theory and Public Opinión", publicado en Public Opinion Quarterly (Berelson, 1952: 313-330). Ambos textos constituyen un ejemplo de la confrontación directa que el autor establece entre los resultados de la investigación empírica y la teoría clásica de la opinión pública. Confrontación en la que Berelson se decantó a favor de la primera.

\section{REFERENCIAS BIBLIOGRÁFICAS}

- Barton, A. (2001): "Paul Lazarsfeld as institutional inventor", International Journal of Public Opinion Research, 13, 3.

- Berelson, B. (1952): "Democracy Theory and Public Opinion", Public Opinion Quarterly, 16, 3.

- (1959): "The State of Communication Research", Public Opinion Quarterly, 23, 1.

- Chaffe, S. H. y J. L. Hochheimer (1985): "The beginnings of political communication research in the United States: origins of the "limited effects model", en Gurevith, M. y Levy, M. R.: Mass Communication Review Yearbook, vol. 5, pp. 75-104.

- Coleman, J. (1982): "Introduction", en KENDALL, P. (ed): The variedad sociology of Paul Lazarsfeld. Writting collected, New York: Columbia University Press.

- Converse, J. M (1987): Survey research in the United States, Los Angeles: University of California Press.

- Glock, C. Y. (1979): "Organizational innovation for social science. Research and training", en Merton, Coleman y Rossi (eds): Qualitative and Quantitative Social Resarch. Papers in honor of Paul F. Lazarsfeld, New York: The Free Press, pp. 19-23. 
- Hyman, H. (1991): Society's Measure: A personal History of Survey Research, New York: Rusell Sage Foundation.

- Katz, E. (1987): "Communication Research since Lazarsfeld" en, Public Opinion Quarterly, 51, 4.

- Lazarsfeld, P. F. (1940): "Panels Studies", Public Opinion Quarterly, 4, 1.

- (1948): "The use of Panel in Social Researh", Proceeding of the American Philosophical Society, 92. Reimpreso en Lazarsfeld, P. F., Pasanella, A. y Rosemberg, M. (eds.) (1972): Continuities in the Languaje of Social Research, New York: The Free Press.

- (1963): "Political Behavior and Public Opinion", en Berelson, B. (ed.): The Behavioral Sciences Today, New York: Basic Books.

- (1972): Qualitative Analysis. Historical and Critical Essays, Boston: Allyn and Bacon Inc.

- Fiske (1938): "The Panel as a new tool for measuring", Public Opinion Quarterly, 2, 4.

- Berelson, B. y Gaudet, H. (1944): The People's Choice, New York: Duell Sloan and Pearce. Segunda Edición, 1948 (con nuevo Prefacio), New York: Columbia University Press. Tercera Edición, 1968 (con nuevo Prefacio), New York: Columbia University Press. En el texto las citas y referencias están tomadas de la tercera edición.

- Merton (1948): "Mass Communication, popular taste, and organized social action", en Bryson, L. (ed): Communication of Ideas, New York: Institute for Religious and Social Studies. Reproducido en, Schramm, W. (1971): The Process and Effects of Mass Communication, Urbana Illinois: University of Illinois Press, pp. 554-578. Traducción al español, Moragas, M. (Ed) (1979): Sociología de la comunicación de masas, Barcelona: Gustavo Gili, pp. 137-157.

घ Rogers, E. M. (1994): A History of Communication Study. A Biografical Approach, New York: The Free Press.

- Rospir, J. I. (1991): "Comunicación política y procesos electorales" en, Muñoz-Alonso et alt.: Opinión Pública y Comunicación Política, Madrid: Eudema, pp. 368-399.

- Schramm, W. (1997): The Beginnings of Communication Study in America. A Personal Memoir, en Chaffe, S. H. y Rogers, E. M. (eds.), California: SAGE.

- Smith, R. B. (2001): "A legacy of Lazarsfeld: cumulative social research on Voting", International Journal of Public Opinion Research, 13, 3.

- Splichal, S. (1989): Public Opinion, New York: Rowman and Littlefield Pbs., Inc. 